Louisiana State University

LSU Digital Commons

Faculty Publications

Department of Physics \& Astronomy

$11-5-2019$

\title{
Organophilic, Superparamagnetic, and Reversibly Thermoresponsive Silica-Polypeptide Core-Shell Particles
}

\author{
Sibel Turksen-Selcuk \\ Louisiana State University \\ Cornelia Rosu \\ Georgia Institute of Technology \\ Alyssa Blake \\ Georgia Institute of Technology \\ Erick Soto-Cantu \\ Louisiana State University \\ Jianhong Qiu \\ Louisiana State University
}

See next page for additional authors

Follow this and additional works at: https://digitalcommons.Isu.edu/physics_astronomy_pubs

\section{Recommended Citation}

Turksen-Selcuk, S., Rosu, C., Blake, A., Soto-Cantu, E., Qiu, J., Wu, Y., Ditusa, J., Steffens, A., \& Russo, P. (2019). Organophilic, Superparamagnetic, and Reversibly Thermoresponsive Silica-Polypeptide Core-Shell Particles. Langmuir, 35 (44), 14248-14257. https://doi.org/10.1021/acs.langmuir.9b01931

This Article is brought to you for free and open access by the Department of Physics \& Astronomy at LSU Digital Commons. It has been accepted for inclusion in Faculty Publications by an authorized administrator of LSU Digital Commons. For more information, please contact ir@lsu.edu. 


\section{Authors}

Sibel Turksen-Selcuk, Cornelia Rosu, Alyssa Blake, Erick Soto-Cantu, Jianhong Qiu, Yan Wu, J. F. Ditusa, Amanda Steffens, and Paul S. Russo 
September 30, 2019

Organophilic, Superparamagnetic and Reversibly Thermoresponsive Silica-Polypeptide Core-Shell Particles

Sibel Turksen-Selcuk, ${ }^{1, \#}$ Cornelia Rosu ${ }^{2,5, \#}$ Alyssa Blake, ${ }^{4 \#}$ Erick Soto-Cantu, ${ }^{1}$ Jianhong Qiu, ${ }^{1}$ Yan Wu, ${ }^{3}$ J. F. DiTusa, ${ }^{3}$ Amanda Steffens ${ }^{l}$ and Paul S. Russo ${ }^{1,2,4 *}$

${ }^{1}$ Department of Chemistry and Macromolecular Studies Group, Louisiana State University, Baton Rouge, Louisiana 70803, USA

${ }^{2}$ School of Materials Science and Engineering and Georgia Tech Polymer Network, Georgia Institute of Technology, Atlanta, Georgia 30332, USA

${ }^{3}$ Department of Physics and Astronomy, Louisiana State University, Baton Rouge, Louisiana, 70803, USA

${ }^{4}$ School of Chemistry and Biochemistry, Georgia Institute of Technology, Atlanta, Georgia 30332, USA

5 School of Chemical and Biomolecular Engineering, Georgia Institute of Technology, Atlanta, Georgia, 30332, USA

${ }^{\#}$ These authors contributed equally to the work.

*To whom correspondence should be addressed:paul.russo@mse.gatech.edu 


\section{ABSTRACT}

Particles with a superparamagnetic cobalt inner core, silica outer core, and covalently bound homopolypeptide shell were investigated under thermal and magnetic stimuli. The homopolypeptide was poly(e-carbobenzyloxy-L-lysine), PCBL, which is known to exhibit a thermoreversible coil $\Leftrightarrow$ helix transition when dissolved as a pure polymer in $m$ cresol. Tethering to a core particle did not prevent PCBL from undergoing this conformational transition, as confirmed by dynamic light scattering and optical rotation, but the transition was broadened compared to that of the untethered polymer. The $\mathrm{Co} @ \mathrm{SiO}_{2}-\mathrm{PCBL}$ hybrid particles retained the superparamagnetic properties of the cobalt inner nougat. Indeed, some response remains even after aging for $>5$ years. The aged PCBL shell also preserved its responsiveness to temperature, although differences in the shape of the size vs temperature transition curve were observed compared to the freshly made particles. A reversible coil $\Leftrightarrow$ helix transition for a particle-bound polypeptide in a pure organic solvent is rare. In addition to providing a convenient tool for characterizing coil $\Leftrightarrow$ helix transitions for surface-bound polypeptides without interference from $\mathrm{pH}$ or the strong ionic forces that dominate behavior in aqueous systems, the $\mathrm{Co} @ \mathrm{SiO}_{2}$ $\mathrm{PCBL} / m$-cresol system may prove useful in studies of the effect of shell polymer conformation on colloid interactions. The stability of the magnetic core and polypeptide shell suggest a long shelf life for $\mathrm{Co} @ \mathrm{SiO}_{2}-\mathrm{PCBL}$, which can in principle be deprotected to yield positively charged $\mathrm{Co}_{0} \mathrm{SiO}_{2}$-poly(L-lysine) particles for possible transfection or antimicrobial applications, or chained magnetically to produce responsive poly(colloids). 


\section{INTRODUCTION}

Interest in stimulus-responsive core-shell particles with tailored structure and surface properties is driven by the demand for such materials in biomedical, electronic, purification and sensory applications. ${ }^{1-3}$ The applied stimulus can be magnetic, electrical, $\mathrm{pH}$, thermal, or photonic, but usually it is just one of these. Multifunctional particles that combine responses are needed to mimic the extraordinary functions of bioparticles. For example, influenza viruses carry information in the form of a nucleic acid "blueprint" for reproduction, along with a shell that expands and contracts in response to $\mathrm{pH}$, helping the particles gain access to the host cell. ${ }^{4}$ Such sophistication is lacking in synthetic particle technology, and this is particularly true for organosoluble particles that can be investigated in the absence of strong salt- or $\mathrm{pH}$-induced effects.

It will be some time before the elegance of bioparticles can be duplicated in the laboratory. Here, we follow a simple approach to combine thermal and magnetic response, along with other features such as chirality and optical activity, by attaching synthetic polypeptides to silica cores to yield polypeptide-composite particles. With only minor variations, silica-polypeptide chemistry can lead to particles that function in aqueous media or typical organic solvents. Polypeptides can exhibit a coil $\Leftrightarrow$ helix transition, even in organic solvents or their mixtures with strong organic acids. ${ }^{5-7}$ This transition tends to be cooperative and sharp. ${ }^{8-9}$ Most of what is known about conformational transitions of covalently bound homopolypeptides has been determined by attaching them to flat surfaces, ${ }^{10-15}$ but polypeptides can be grown one monomer at a time on spherical silica particles, or even attached to them after polymerization. This field has been reviewed, ${ }^{16-17}$ as has recent progress in polypeptide synthesis ${ }^{18}$ and responsiveness. ${ }^{19}$ In addition to drug delivery or protein enrichment, ${ }^{20}$ potential 
applications include colloidal crystals that self-anneal, taking advantage of thermal transitions to grow larger domains for improved performance, or poly(colloids) created by magnetic chaining that expand or contract in response to thermal stimulus. After deprotection of the benzyl sidechain and dispersion in an aqueous medium, such poly(colloid) fibrils can be expected to expand and contract in response to $\mathrm{pH}$ or salt. Long ago, Pauling and Corey considered the coil $\Leftrightarrow$ helix transition as the motive force underlying muscle action. ${ }^{21}$ Today we know that is not how muscles work, but Pauling and Corey's calculation of the energetics shows that it is not unreasonable to dream of muscle-like actuators built by assembling polypeptide composite particles.

To start along a path leading to such futuristic materials, we investigate the reversible coil $\Leftrightarrow$ helix conformational transition of PCBL (poly( $\varepsilon$-carbobenzyloxy-Llysine)) covalently attached to a $\mathrm{Co} @ \mathrm{SiO}_{2}$ core in response to thermal and magnetic stimuli. Unlike the more commonly studied PBLG (poly( $\gamma$-benzyl-L-glutamate)), which has been attached to $\mathrm{Fe}_{3} \mathrm{O}_{4}$-containing magnetic silica particles, ${ }^{22}$ untethered $\mathrm{PCBL}$ polymer exhibits an inverse (coil to helix) transition as the temperature rises above $27^{\circ} \mathrm{C}$ in $m$-cresol. ${ }^{5}$ The transition is fully reversible. From the standpoint of fundamental science, the existence of a well-behaved conformational change in a pure organic solvent is especially attractive because transitions can be studied without interference from strong electrostatic forces. It was known at the outset of this study that particles with a very high grafting density and therefore a crowded surface fail to exhibit a coil $\Leftrightarrow$ helix transition. ${ }^{23}$ The shell cannot be too sparsely populated with PCBL, though, because then its transitions will be difficult to follow. To achieve a response that can be detected, the particles presently under discussion were made using partially functionalized/partially passivated silica cores as described previously. ${ }^{24} \mathrm{~A}$ variety of 
techniques confirm the coil $\Leftrightarrow$ helix transition for the PCBL polymer when tethered to the $\mathrm{Co} @ \mathrm{SiO}_{2}$ particle. The PCBL polymer, analyzed after detachment from the $\mathrm{Co} @ \mathrm{SiO}_{2}$ core, exhibits a remarkably broad molecular weight dispersity. The possibility that the PCBL shell, which greatly outweighs the cobalt inclusion, could modulate the overall particle magnetic response is considered. Portions of this work have appeared in preliminary form. ${ }^{25-27}$

\section{EXPERIMENTAL SECTION}

General Synthetic Procedures. The overall preparation of the particles is shown in Scheme 1. Unless otherwise noted, all synthetic procedures were carried out in a dry, inert nitrogen atmosphere. Chemicals and solvents were obtained from Sigma-Aldrich, Pharmco-AAPER, or Acros Organics with the highest purity available, as described in greater detail elsewhere, ${ }^{28-31}$ and used without further purification. The solvents used for the synthesis of the monomers and covalently attached polymer shell were all anhydrous and purchased in Aldrich Sure-Seal ${ }^{\mathrm{TM}}$ bottles. Water was supplied by a Barnstead Nanopure Water System (18 M $\Omega \mathrm{cm}$ ) equipped with spiral-wound ultrafilter (nominally $5 \mathrm{~nm})$.

\section{Synthesis of superparamagnetic cobalt coated with silica, $\mathrm{Co} @ \mathrm{SiO}_{2}$, and amino-} functionalized silica-coated cobalt, $\mathbf{C o} @ \mathbf{S i O}_{2}-\mathbf{N H}_{2}$. The procedure to synthesize the superparamagnetic cores and functionalize their surfaces is based on a method developed by Kobayashi et al., ${ }^{32}$ with some modifications. ${ }^{33}$ A solution consisting of $200 \mathrm{~mL}$ of $4.0 \times 10^{-3} \mathrm{M} \mathrm{NaBH}_{4}$ was prepared with $4.0 \times 10^{-4} \mathrm{M}$ citric acid. While sonicating and bubbling with nitrogen, $200 \mathrm{~mL}$ of $\mathrm{NaBH}_{4}$ was mixed with $0.2 \mathrm{~mL}$ of 0.4 $\mathrm{M} \mathrm{CoCl}_{2} \cdot 6 \mathrm{H}_{2} \mathrm{O}$ for $1 \mathrm{~min}$ and $800 \mathrm{~mL}$ of absolute ethyl alcohol solution containing 14.4 $\mu \mathrm{L}$ 3-aminopropyltrimethoxysilane (APTMS) and $169 \mu \mathrm{L}$ tetraethylorthosilicate (TEOS) 
was added rapidly. The reaction continued for $1 \mathrm{~h}$ under $\mathrm{N}_{2}$ bubbling and probe sonication. After $1 \mathrm{~h}$ the particles were collected by centrifugation and washed $3 \times$ with anhydrous ethanol. The collected particles were dispersed in $500 \mathrm{~mL}$ of absolute ethyl alcohol and mixed for $18 \mathrm{~h}$ with $0.1 \mathrm{~mL}$ of a mixture of $75 \%$ methyl trimethoxysilane (MTMS) and 25\% N-[3-(Trimethoxysilyl)propyl]ethylenediamine] (AEAPTMS for the traditional, non-IUPAC name [(aminoethylamino)propy]ltrimethoxysilane). Afterwards, the mixture was concentrated to $1 / 3$ of its original volume by a rotary evaporator. Then the particles were washed by centrifugation-redispersion with anhydrous ethanol until a ninhydrin test indicated proved the absence of free amine groups in the decanting liquid.

\section{Preparation of Ne-carbobenzyloxy-L-lysine N-carboxyanhydride (CBL-NCA)}

monomer. The general procedure of Daly and Poche' ${ }^{34}$ was used, including the product purification method of Poché. ${ }^{40}$ In a typical preparation, 5 grams of the amino acid $\mathrm{N} \varepsilon$ carbobenzyloxy-L-lysine (CBL) was dissolved in $150 \mathrm{~mL}$ of anhydrous ethyl acetate in a 3-neck round bottom flask fitted with a reflux condenser and a nitrogen bubbler. The solution was heated to reflux and no more than $1 / 3$ equivalent of triphosgene was added rapidly. The reaction continued to reflux under nitrogen for $4 \mathrm{~h}$. As the reaction solution cleared, the flask containing the mixture was cooled to $-5^{\circ} \mathrm{C}$ overnight. The cold solution was then washed quickly in a separatory funnel with $50 \mathrm{~mL}$ de-ionized water cooled to $0^{\circ} \mathrm{C}$, followed by a wash with $50 \mathrm{~mL}$ of $0.5 \% \mathrm{NaHCO}_{3}$ chilled to $0^{\circ} \mathrm{C}$. The organic layer was dried with anhydrous $\mathrm{MgSO}_{4}$ as quickly as possible and gravity filtered. The solution was concentrated to $1 / 3$ of its original volume under constant flow of nitrogen in a glove bag. Afterwards, $100 \mathrm{~mL}$ of anhydrous hexane was added to the solution causing the precipitation of the monomer. The concentrated slurry was kept overnight at 
$-5^{\circ} \mathrm{C}$, then collected by suction filtration in a glove bag under constant flow of nitrogen. The CBL-NCA monomer was dried in a vacuum oven for $24 \mathrm{~h}$. The typical yield is 70\%. Additional details appear elsewhere. ${ }^{35}$

Polymerization of untethered poly( $\varepsilon$-carbobenzyloxy-L-lysine), PCBL. In nitrogenfilled glove bag, $\sim 2.00 \mathrm{~g}$ of CBL-NCA was dissolved in $50 \mathrm{~mL}$ of anhydrous THF inside a $100 \mathrm{~mL}$ oven-dried round bottom flask. A magnetic stir bar was placed into the flask and capped with a rubber septum. The flask was placed into an aluminum bead bath (Lab Armor Beads) and sodium methoxide solution $(0.3 \mathrm{~mL})$ was injected. A bubbler containing silicon oil was connected to the flask and the reaction was heated to $35{ }^{\circ} \mathrm{C}$ and stirred until there was no bubble formation inside the oil in the bubbler (usually 2 days). The flask was then connected to a rotatory evaporator to red uce volume to $1 / 3$ to obtain a viscous solution that was slowly poured into a beaker containing water. This caused the polypeptide to precipitate out of solution and the precipitate was suction filtered using a Whatman $90 \mathrm{~mm}$ filter paper and allowed to dry.

Synthesis of $\mathrm{Co} @ \mathrm{SiO}_{2}-\mathrm{PCBL}$ particles by growing-from procedure. The aminofunctionalized $\mathrm{Co} @ \mathrm{SiO}_{2}-\mathrm{NH}_{2}$ particles prepared as described above were dispersed in anhydrous dioxane by centrifugation-redispersion $(2 \times)$. Then typically $20 \mathrm{~mL}$ of the dispersion was added to $5.0 \mathrm{~g}$ of the CBL-NCA, which was pre-dissolved in $200 \mathrm{~mL}$ of anhydrous dioxane in a round-bottom flask. The reaction vessel was connected to an 
Erlenmeyer flask filled with $50 \mathrm{~mL} 1 \mathrm{~N} \mathrm{NaOH}$ to capture the released $\mathrm{CO}_{2}$. The reaction continued for 3 days at $35{ }^{\circ} \mathrm{C}$ in a nitrogen-inflated glove bag. The particles were collected by centrifugation and washed three times with dioxane and three times with dimethylformamide (DMF) to remove any untethered PCBL chains. In previous studies, it was demonstrated that deliberately added polypeptide chains were removed by a similar procedure, while attached chains remain. ${ }^{28}$ The XPS spectra confirm the desired chemistry at key steps (Figures S3 and S4). The particle batch was split and finally dispersed in DMF and $m$-cresol.

Removal of $\mathrm{Co} @ \mathrm{SiO}_{2}$ Core. $\mathrm{Co} @ \mathrm{SiO}_{2}-\mathrm{PCBL}$ particles were precipitated out of DMF suspensions by adding water. The sample in a 4-mL vial was placed into a vacuum oven overnight at $T<60^{\circ} \mathrm{C}$ to remove the water completely. The dried $\mathrm{Co} @ \mathrm{SiO}_{2}-\mathrm{PCBL}$ particles had a mass of $\sim 5-10$ milligrams. About $2 \mathrm{~mL}$ of $5 \% \mathrm{HF}$ solution was added to the dried particles (Caution: HF is toxic; double gloves, face shield, lab coat and apron are required, as well as calcium gluconate set aside from the immediate work space to apply to skin in case of contact. We additionally worked in teams of at least two.) The vials sat undisturbed for 48 hours, allowing the HF to penetrate the particles and etch the silica cores. The 5\% HF solution was then removed from the vial using a disposable pipet, leaving the solid polymer in the vial. The now-untethered polypeptide was washed $3 \times$ with clean water and then placed into a vacuum oven overnight to dry.

Dynamic Light Scattering. Samples were prepared as described in the Supporting Information. A custom-built multiangle apparatus equipped with $632.8 \mathrm{~nm}$ laser and ALV-5000 digital autocorrelator was used. To assess particle uniformity at three temperatures, measurements were made at multiple scattering angles from $30^{\circ}$ to $90^{\circ}$. 
Subsequently, details of the expansion and contraction of $\mathrm{Co} @ \mathrm{SiO}_{2}-\mathrm{PCBL}$ particle size in $m$-cresol were monitored with greater temperature resolution for several heatingcooling cycles at a single scattering angle of $75^{\circ}$. Cumulants and single-exponential analyses were used to fit the autocorrelation functions for these particles. Using the Stokes-Einstein relation, the apparent hydrodynamic radius, $R_{\mathrm{h}}$,app was calculated from the apparent diffusion, $D_{\text {app }}$, defined as the autocorrelation function decay rate $\Gamma$ divided by the squared scattering vector magnitude, $q^{2}$. The required $m$-cresol viscosity $(\eta)$ data as a function of Kelvin temperature $(T)$ were taken from Tewari, Vesudevan and Ramakrishna. ${ }^{36}$ Their results can be summarized by a linear Arrhenius fit:

$\ln (\eta / \mathrm{cP})=(-13.20 \pm 0.23)+(4709.7 \pm 67.3) \times(T / \mathrm{K})^{-1}$

Transmission Electron Microscopy (TEM). Samples were deposited onto carboncoated copper specimen grids and dried in air. A Hitachi HD-2700 STEM working at an accelerating voltage of $200 \mathrm{kV}$ was used to image the individual silica-coated cobalt particles. A JEOL $100 \mathrm{CX}$-II TEM working at an accelerating voltage of $100 \mathrm{kV}$ was used to image the larger grouping of silica-coated cobalt particles and the silica coated cobalt snakes.

Scanning Electron Microscopy (SEM). Samples were deposited onto silicon wafers and dried in air. A Hitachi SU8230 SEM working at an accelerating voltage of $1.5 \mathrm{kV}$ was used to image the non-magnetic polypeptide-coated silica particles using the lower secondary electron detector. The SEM working at an accelerating voltage of $5 \mathrm{kV}$ was used to image the polypeptide-coated cobalt-silica particles using both the upper and lower second ary electron detectors. 
Gel Permeation Chromatography/Multiangle Light Scattering (GPC/MALS). The molecular weight of PCBL was measured using a Tosoh EcoSEC HLC-8320GPC connected to a Wyatt Dawn EOS multi-angle-light scattering detector. The mobile phase was $\mathrm{N}, \mathrm{N}$-Dimethylformamide with $0.1 \mathrm{M} \mathrm{LiBr}$ flowing at $0.35 \mathrm{~mL} / \mathrm{min}$. A TSK guard column SuperH-H $(4.6 \mathrm{~mm}$ ID $\times 3.5 \mathrm{~cm} \mathrm{~L})$ was placed before two TSKgel SuperHM-H (mixed-bed) analytical columns $(6.0 \mathrm{~mm} \mathrm{ID} \times 15 \mathrm{~cm} \mathrm{~L})$, and the instrument temperature was set to $60{ }^{\circ} \mathrm{C}$. Polymer samples of concentration $3-8 \mathrm{mg} / \mathrm{mL}$ were dissolved overnight prior to measurements. Solutions were filtered through Whatman PTFE 0.45 $\mu \mathrm{m}$ syringe filters into Agilent $2 \mathrm{~mL}$ GPC vials containing a $250 \mu \mathrm{L}$ borosilicate glass conical insert to accommodate the small total volume. Three injections $(20 \mu \mathrm{L})$ were performed for each sample. The specific refractive index increment, $d n / d c$, was 0.114 $\mathrm{mL} / \mathrm{g}$.

X-Ray Photoelectron Spectroscopy (XPS). The surface composition of the core and core-shell particles was investigated with a Kratos Analytical Axis 165 X-ray photoelectron spectrometer (XPS) with Al Ka-radiation with an energy of $1.48 \mathrm{keV}$ and a takeoff angle of $90^{\circ}$. For each sample, a survey spectrum was acquired using a pass energy of $80 \mathrm{eV}$, in addition to high-resolution spectra of all individual elements using a pass energy of $40 \mathrm{eV}$. The peak locations were calibrated based on the $\mathrm{C} 1 \mathrm{~s}$ signal at 284 $\mathrm{eV}$.

Optical Rotation. Optical rotation measurements were carried out on a Jasco CD J-815 spectropolarimeter equipped with an ORDM-401 attachment featuring an optical null. Instrument performance was verified using sucrose in water and poly $(\gamma$-benzyl- $\alpha, \mathrm{L}-$ 
glutamate) in dimethylformamide, DMF. ${ }^{6,8,37-38}$ The optical rotation of PCBL-coated cobalt particles was measured in a temperature range of $10-50{ }^{\circ} \mathrm{C}$ with a precision of \pm $0.1{ }^{\circ} \mathrm{C}$. Each reported spectrum is a collection of 5 accumulations consisting of wavelength scans from $600-300 \mathrm{~nm}$. Specific optical rotation at $436 \mathrm{~nm}$ was calculated from the raw optical rotation and subtracted manually from the blank (a cell containing $m$-cresol). An ORDM ZeroClear protocol was executed before each sample was analyzed. The raw optical rotation data was converted into specific optical rotation using the equation,

$$
\alpha_{s p}=\frac{100 \cdot \alpha_{o p}}{l \cdot c}
$$

where $\alpha_{\mathrm{sp}}$ is the specific optical rotation, $\alpha_{\mathrm{op}}$ is optical rotation, $l$ is the length of the cuvette in $\mathrm{dm}$, and $c$ is the concentration of the solution in $\mathrm{g} / 100 \mathrm{~mL}$.

Fourier Transform Infrared Spectroscopy (IR). Infrared spectra were recorded with a Bruker Tensor 27 FT-IR spectrometer. A Spectra Tech high-pressure in situ reaction cell with a $\mathrm{ZnSe}$ ATR crystal was used to monitor the spectral changes of $\mathrm{Co} @ \mathrm{SiO}_{2}-\mathrm{PCBL}$ in $m$-cresol as a function of temperature. Spectra were analyzed with OPUS 4.2 software. Each spectrum is a collection of 16 scans depending on the sample concentration ( 50-60 ppm). Solvent ( $m$-cresol) was measured as a background before the sample measurements.

Magnetization and Magnetic Susceptibility. Magnetic properties of the particles were measured in a Quantum Design Magnetic Property Measurement System (MPMS) SQUID magnetometer for temperatures between 10 and $50^{\circ} \mathrm{C}$ in fields of up to $50 \mathrm{kOe}$. Dried $\mathrm{Co} @ \mathrm{SiO}_{2}$ samples were placed in gelatin capsules and measured inside quartz 
tubes. Liquid $\mathrm{Co} @ \mathrm{SiO}_{2}-\mathrm{PCBL}$ dispersions were measured in custom-built quartz tubes with a seal made of poly(tetrafluoroethylene). Empty cell backgrounds were measured and subtracted from the raw data.

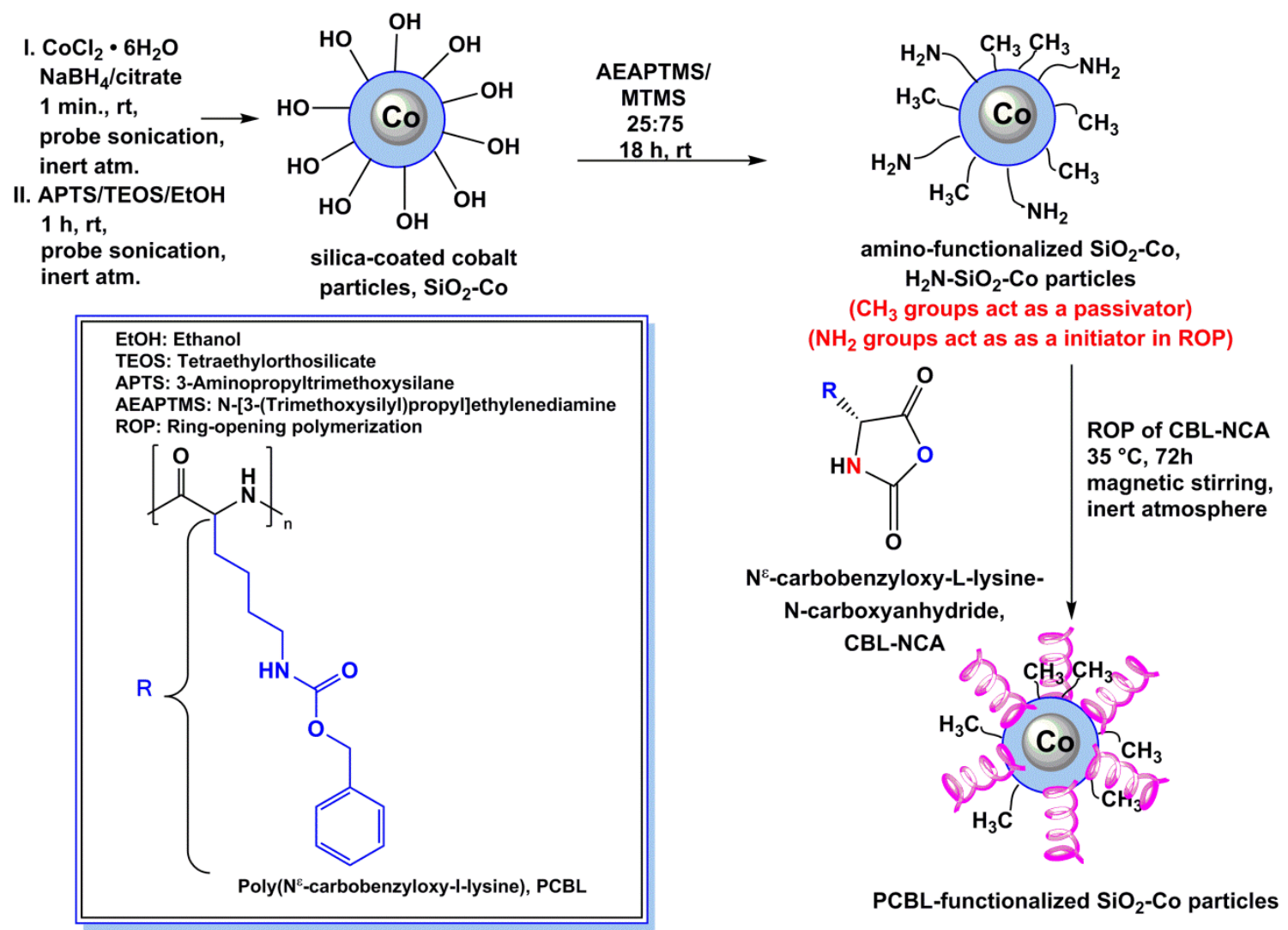

Scheme 1. Schematic illustration of the steps involved in the preparation of PCBLfunctionalized superparamagnetic silica particles $\left(\mathrm{Co} @ \mathrm{SiO}_{2}-\mathrm{PCBL}\right)$.

\section{RESULTS AND DISCUSSION}

Particle synthesis. Scheme 1 illustrates the steps involved in the preparation of the $\mathrm{Co} @ \mathrm{SiO}_{2}-\mathrm{PCBL}$ particles. After the cobalt-containing silica particles were synthesized, their surface was functionalized with a mixture of MTMS (75\%) as spacer group and 
surface passivator and AEAPTMS (25\%) as initiator for the ring-opening polymerization of CBL-NCA monomer.

Particle Visualization. TEM images of typical cobalt-containing core particles are shown in Figure 1A. Most particles have a single cobalt inclusion, but imperfections do occur during the synthesis. Particles lacking a Co inclusion (a few are visible in the inset to Figure 1A) are easily separated from the Co-containing ones by attraction of the latter towards a magnet. A different issue can arise if the Co inclusion grows too large. If the diameter of the cobalt particles exceeds about $8-10 \mathrm{~nm}$, permanent magnetic dipoles become stable and the particles chain together even without application of a magnetic field (Supporting Information, Figure S1). Such particles were not used in this experiment but isolated and retained for other applications. Figure 1B shows a SEM image of the polypeptide-coated magnetic silica recovered after scattering measurements in $m$-cresol, followed by solvent exchange to DMF before deposition on the SEM stub. The inset shows a larger count of PCBL-coated silica particles, in this case without the magnetic nougat to demonstrate that magnetic and non-magnetic particles have the same spherical shape and size.

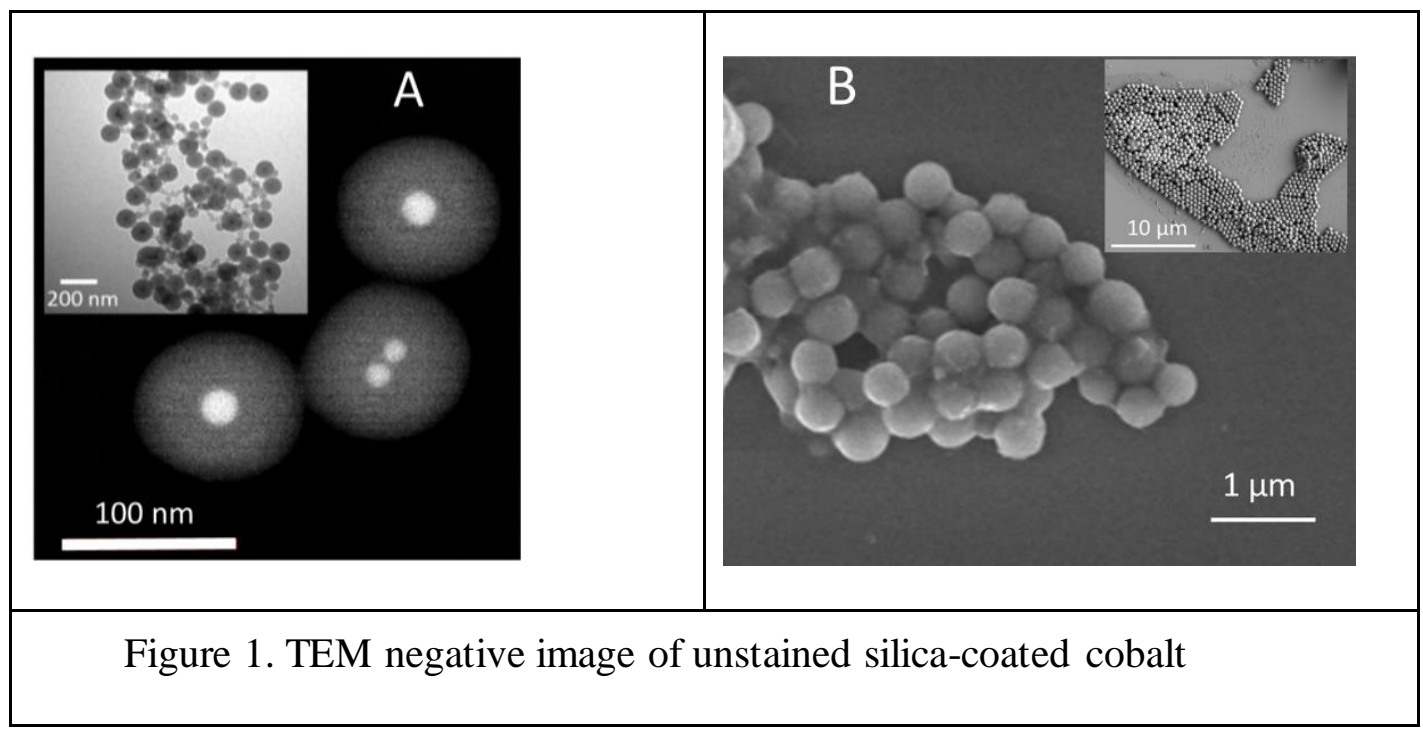


particles; most have one central Co inclusion; inset shows positive image prior to isolation of Co-containing particles by magnetic separation (A). SEM image of $\mathrm{Co} @ \mathrm{SiO}_{2}-\mathrm{PCBL}$ composite particles; inset: nonmagnetic $\mathrm{PCBL}-\mathrm{SiO}_{2}$ particles (B).

Electron diffraction patterns were featureless, due to the amorphous nature of cobalt and silica, in agreement with Kobayashi et al. ${ }^{39}$

Particle Characteristics. The batch chosen for this study had a $\mathrm{Co} @ \mathrm{SiO}_{2}$ core radius of $88 \mathrm{~nm}$ and Co inclusion radius of $\sim 9 \mathrm{~nm}$. X-ray photoelectron spectra (Figures S3 and S4) measured at various stages during the synthesis and subsequent thermogravimetric analysis of the final product demonstrated the progression of surface functionality expected for the reactions in Scheme 1. Primary amines do normally initiate NCA monomers with attachment, ${ }^{40}$ but absolute proof that the initiating amines have done so in this case is difficult for high- $M$ shells. Previous studies have demonstrated the effectiveness of the washing procedure, though. It completely removes unbound polypeptide even after its deliberate addition to bare silica. ${ }^{28}$ Thermogravimetric analysis (Figure S5) quantified the amount of shell material present. The PCBL shell on the samples selected for further investigation accounted for $\sim 39 \%$ of particle mass. As shown in Supporting Information, this indicates well-solvated chains. The percentage of mass attributed to the Co nougat is miniscule by comparison, about $0.3 \%$ of the total particle mass, about 130 times less than the PCBL mass (Figure S5 and analysis in Supporting Information). 
After digestion of the core by HF and collection of the PCBL shell and dissolution into the DMF-LiBr mobile phase, GPC/MALS revealed predominantly low$M$ polymer $\left(2,700 \mathrm{~g} \cdot \mathrm{mol}^{-1} ; Ð=1.1\right)$ but with substantial amounts of high- $M$ polymer $\left(M_{\mathrm{w}}=96,500 \mathrm{~g} \cdot \mathrm{mol}^{-1} ; Ð=1.7\right)$. The weight average length of the larger polymer in helix form is $L_{\mathrm{w}}=55 \mathrm{~nm}$ (i.e., $M_{\mathrm{w}} \times h / M_{\mathrm{o}}$ where $h$ is the axial projection of the $\alpha$-helix, 0.15 $\mathrm{nm}$, and $M_{\mathrm{o}}$ is the monomer molar mass, $263 \mathrm{~g} \cdot \mathrm{mol}^{-1}$ for PCBL). Figure 2 reveals the presence of even larger polymers, up to at least $M=400,000 \mathrm{~g} \cdot \mathrm{mol}^{-1}$, corresponding to $L$ $=230 \mathrm{~nm}$. When added to the $\mathrm{Co} @ \mathrm{SiO}_{2}$ core $(88 \mathrm{~nm}$ radius $)$, the complete particle radius is expected to be about $318 \mathrm{~nm}$ at full helix extension. This estimation assumes possible extension of the helix near the particle surface is offset by partial draining near the particle periphery.

Figure 2B shows triplicate runs of the measured radius of gyration, $R_{\mathrm{g}}$, of the PCBL chains clipped from the particles by HF, overlaid on triplicate runs of nevertethered PCBL prepared by initiation in free solution. The traces for the never-tethered PCBL are noisier because this sample was more uniform in length, meaning that high- $M$ and low- $M$ data lie in the wings of a GPC/MALS peak that is narrow compared to that shown in Figure 2A. Neither polymer approaches the sizes achieved by Fasman, Idelson and Blout ${ }^{41}$ using methoxide as initiator, but they can be compared in the molecular weight range spanning 100,000 to $200,000 \mathrm{~g} \cdot \mathrm{mol}^{-1}$. In this range, the HF-clipped PCBL and never-tethered PCBL have about the same $R_{\mathrm{g}}$ values, so the physical attributes, as seen by light scattering, are the same. Neither PCBL sample matches the expected size for a completely rigid $\alpha$-helix $\left(R_{\mathrm{g} \text {,helix }}=L / 12^{1 / 2}\right.$ where $\left.L=M \times h / M_{\mathrm{o}}\right)$ but this is not surprising, as PCBL has long been known to lack the rigidity of, say, PBLG. ${ }^{41}$ The data are consistent with a Flory exponent, $v_{\mathrm{F}}$ in $R_{\mathrm{g}} \propto M^{v_{\mathrm{F}}}$, of about 0.8 . This value should not 
be regarded as definitive for PCBL/DMF- $0.1 \mathrm{M} \mathrm{LiBr}$ because the range of measurement is too small, but it indicates an extended polymer.

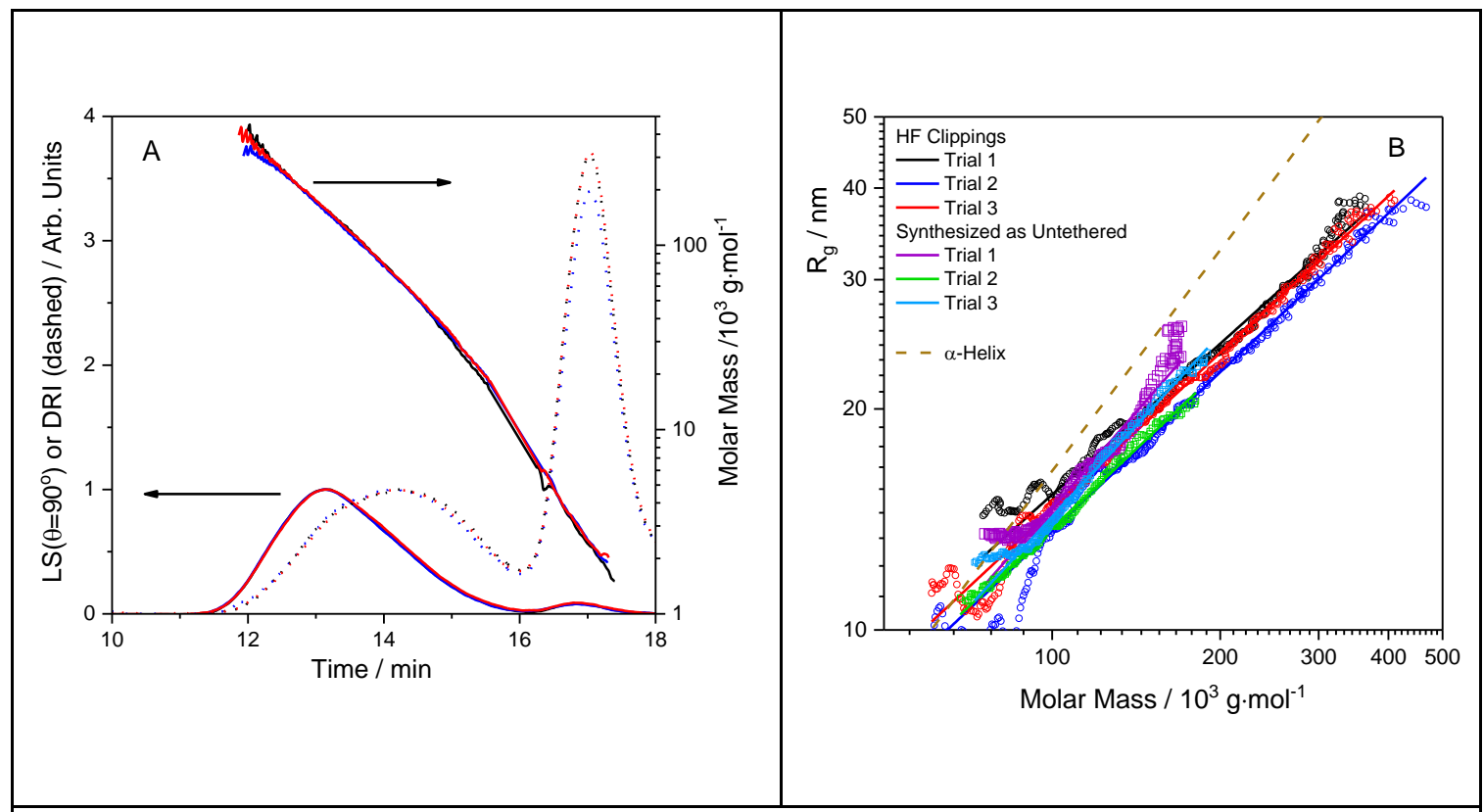

Figure 2. GPC/MALS molar mass chromatograms for PCBL clipped from $\mathrm{Co} @ \mathrm{SiO}_{2-}$ PCBL particles by HF dissolution of the core, together with overlay of light scattering intensity (LS, $\theta=90^{\circ}$ ) and differential refractive index (DRI) signals, triplicate runs (A); Conformation plots of radius of gyration vs molar mass for polymer clipped from $\mathrm{Co} @ \mathrm{SiO}_{2}$-PCBL particles, with overlay plots for never-tethered, methoxide-initiated PCBL, triplicate runs for each polymer (B). 
Conformational Transition-DLS Evidence. DLS was used to follow the temperature-driven conformational change of $\mathrm{Co} @ \mathrm{SiO}_{2}-\mathrm{PCBL}$ composite particles in $m$ cresol, illustrated in Scheme 2.

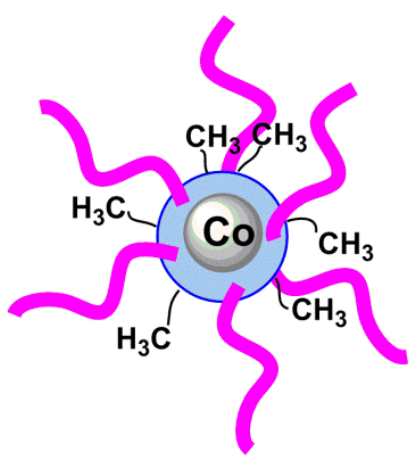

random coil PCBL

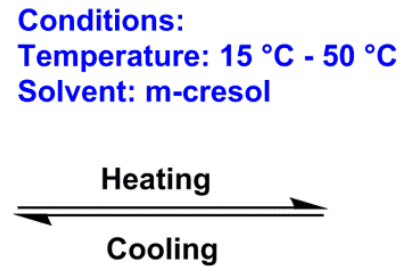

$\alpha$-helical PCBL

Scheme 2. Schematic illustration of the conditions driving the inverse coil to helix transition of PCBL in $m$-cresol as a function of temperature. Not to scale.

To bracket the response of the system, multiple-angle measurements were made at three temperatures, 15,25 , and $50{ }^{\circ} \mathrm{C}$, values that lie below, near and above the known transition of untethered PCBL $\left(\sim 27^{\circ} \mathrm{C}\right)$. The results (Figure 3) show an increase in apparent hydrodynamic radius, $R_{\mathrm{h}}$,app, with temperature. The apparent radii meet the expectations of the above section and do not depend significantly on scattering angle, which for large particles is evidence that the suspensions are afflicted neither by aggregation nor by broad particle size distribution. 


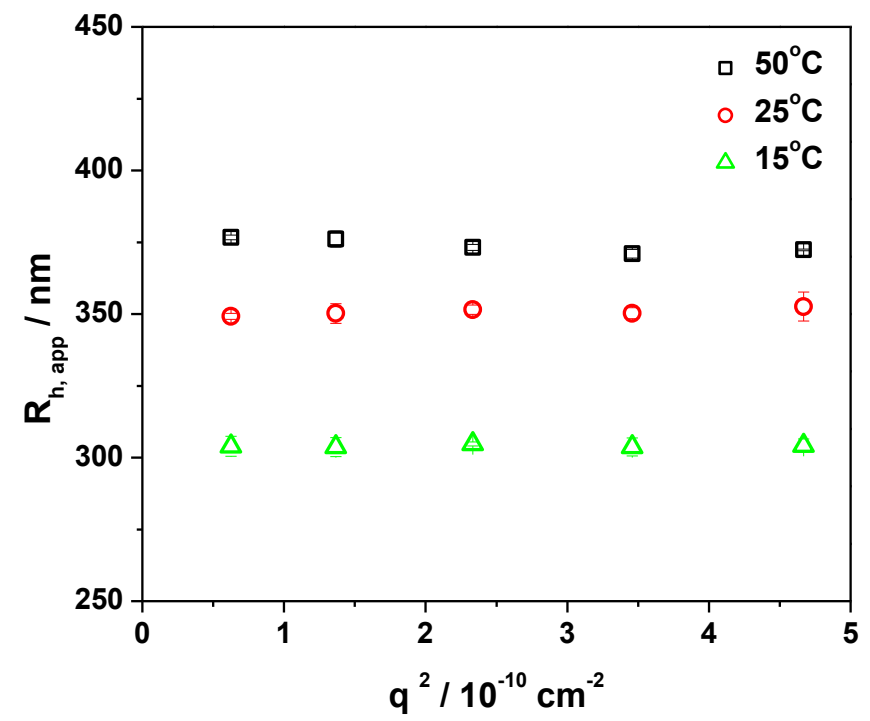

Figure 3. Apparent hydrodynamic radius of the $\mathrm{Co} @ \mathrm{SiO}_{2}-\mathrm{PCBL}$ particles measured at 15,25 , and $50^{\circ} \mathrm{C}$ in $m$-cresol plotted at different squared scattering vector magnitudes, corresponding to different scattering angles.

The independence of $R_{\mathrm{h} \text {,app }}$ on scattering angle justifies the use of a single scattering angle for studies at additional temperatures. A $\mathrm{Co} @ \mathrm{SiO}_{2}-\mathrm{PCBL}$ dispersion in $m$-cresol was measured at a scattering angle of $75^{\circ}$ over three heating-cooling cycles in the $10-50^{\circ} \mathrm{C}$ temperature range. At each temperature step, the sample was equilibrated for 30 minutes before acquiring DLS data. This well exceeds instrument response time (<10 min). As shown in Figure 4, the particle shells expand on heating. This response is attributed to the coil $\Leftrightarrow$ helix transition of the PCBL shell, in consideration of the known behavior of untethered PCBL in $m-\mathrm{cresol}^{5}$ and the knowledge that only small polypeptides fail to expand when the helix is formed. ${ }^{42}$ The PCBL shell shrinks on cooling, corresponding to return to the random coil configuration. On the first heating cycle, the size is $\sim 5 \%$ smaller than reported in Figure 3; such a difference is not cause for great concern, but it does suggest future studies might benefit from even longer 
equilibration times between temperature steps. Also, the $20 \mathrm{~nm}$ downward drift between the end of the first heating cycle and start of the first cooling cycle suggests the particles may "overshoot" their size expansion at first. The behavior grew erratic (slightly different low- and high-temperature radii) after the third heating cycle, but transitions were still observed. Neither the "overshoot" nor the erratic behavior after three cycles is understood. The normalized variance from the cumulant fits, ${ }^{43} \mu_{2} / \Gamma^{2}$, remained low (average value 0.05) and angle-invariant over the temperature range. This result, along with the above-noted independence of $R_{\mathrm{h}}$, app with scattering angle, argue against temperature induced aggregation as an explanation of the reversible transition seen in Figure 4.

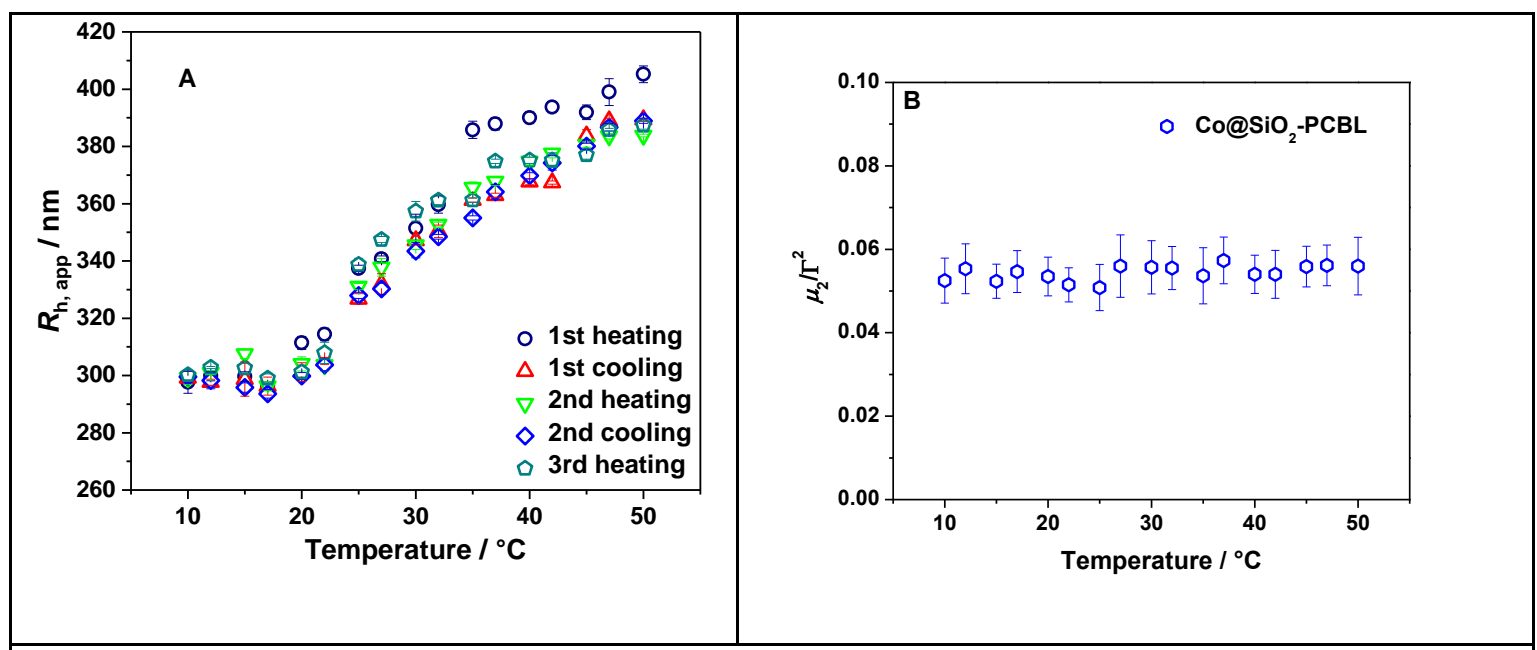

Figure 4. DLS results of PCBL-coated cobalt particles in $m$-cresol: (A) Temperature dependence of apparent hydrodynamic radius of PCBL particles (error bars represent triplicate runs at a single scattering angle, $75^{\circ}$ ) and (B) corresponding $\mu_{2} / \Gamma^{2}$ vs. temperature plot; each point is average of data collected during all heating and cooling cycles. 
Neither did the intensity values from static light scattering reveal signs of aggregation. We therefore rule out aggregation as an explanation for the transition or its minor peculiarities, such as overshoot and erratic behavior after three cycles. Investigation of those details would benefit from a method with greater resolution than DLS. Perhaps small-angle neutron scattering performed on particles with shells deuterated at selected sites could reveal more, though the limitations of neutron flux might prove a hardship for time-resolved studies. Meanwhile, the size transition appearing in Figure 4 is regarded as a quasi-equilibrium phenomenon. It is centered at a temperature close to the transitions reported by by Matsuoka et al., who applied various methods to a range of molecular weights of untethered PCBL dissolved in $m$-cresol. ${ }^{5}$ Our transition, which spans at least $20^{\circ} \mathrm{C}$, is broader than was reported at any molecular weight by these authors (their transition spans about $3^{\circ} \mathrm{C}$ for the sample closest in molecular weight to our $M_{\mathrm{w}}$, to judge from Figure 7 of Ref. 5). One possible cause for the broadening is the wide range of molecular weights present on the particle, as revealed by Figure 2B.

Before moving ahead to methods that probe chain conformation more directly than DLS, we note that the possibility was considered that the transitions seen in Figure 4 could arise from the viscosity contribution of small amounts of residual untethered PCBL. As shown in the Supporting Information, the viscosity contribution of any untethered polymers cannot account for the behavior seen in Figure 4, even in a worstcase scenario.

Conformational Transition-Optical Rotation Evidence. The scattering experiments show a size change near the temperature at which untethered PCBL undergoes a coil-tohelix transition in $m$-cresol. In order to explore the nature of the transition, techniques sensitive to molecular conformations were applied. The magnetic character of the 
particles precludes the use of NMR, which is sometimes adept at following conformational transitions. ${ }^{44}$ We prepared a nonmagnetic particle by omitting the nanoscale cobalt; these particles appeared in Figure 1B. The NMR behavior of such particles dispersed in $m$-cresol is best reserved for a separate report. Briefly, although certain peaks shifted by up to $1 \mathrm{ppm}$ throughout the temperature range of interest, it was non-trivial to separate spectral changes due to polypeptide conformational transitions from general drift of the NMR peak positions due to temperature-induced changes in the overall H-bonding environment. NMR lifetime experiments are likely to prove revealing, but they are not necessary for the present purpose of determining whether the size transition in Figure 4 is caused by a change in polypeptide conformation.

In the polypeptide field, optical rotation and circular dichroism spectroscopy are often considered the most reliable indicators of chain conformation. ${ }^{32-36}$ Although circular dichroism is ineffective in UV-opaque solvents including $m$-cresol, optical rotation works well. In our experiments, the spectrometer is set at a single wavelength and operates as a precise polarimeter with automated temperature control. Figure 5 shows optical rotation results for $\mathrm{Co} @ \mathrm{SiO}_{2}-\mathrm{PCBL}$ particles from the same batch used for the DLS measurements, along with results from control experiments on untethered PCBL and PBLG. The response is normalized by the amount of polypeptide present, which must be maintained at a low value in the case of $\mathrm{Co} @ \mathrm{SiO}_{2}-\mathrm{PCBL}$ particles in order to reduce interference from scattered light; these low concentrations account for the noise in the $\mathrm{Co} @ \mathrm{SiO}_{2}-\mathrm{PCBL}$ signal. Early investigators of synthetic polypeptides established that the helical conformation gives rise to a less negative/more positive optical rotation versus the random coil conformation. ${ }^{41}$ Thus, the increase from $-200^{\circ}$ to $-50^{\circ}$ for untethered PCBL indicates a change in the polypeptide structure to $\alpha$-helix at 
higher temperatures. A noisier, yet still distinct, transition from $-200^{\circ}$ to $+25^{\circ}$ is observed for PCBL attached to $\mathrm{Co} @ \mathrm{SiO}_{2}$. This again indicates structural change from random coil to helix, but the transition begins at a lower temperature and the change is larger. Indeed, the specific optical rotation approaches the nearly constant value seen for untethered PBLG, which remains in the helical conformation in $m$-cresol throughout the measured temperature range. The results confirm the temperature dependence reported long $\mathrm{ago}^{5}$ for the optical rotation of untethered PCBL in $m$-cresol, and reveal that the same transition occurs when PCBL is attached to a particle, though broadened, in agreement with the DLS results.

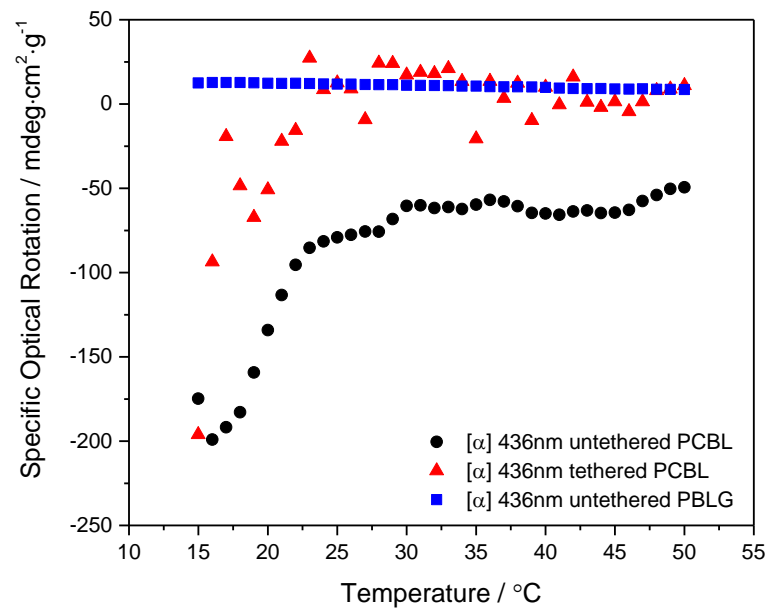

Figure 5. Temperature dependence of the specific optical rotation at $436 \mathrm{~nm}$ of PCBL in m-cresol $(\bullet), \mathrm{Co} @ \mathrm{SiO}_{2}-\mathrm{PCBL}$ in $m$-cresol $(\boldsymbol{\Delta})$, and PBLG in $m$-cresol ( $\square$ ). The data are normalized by the amount of polypeptide.

Conformational Transition-FTIR Evidence. FTIR measurements support the existence of a reversible, temperature-driven coil-to-helix transition for PCBL 
covalently attached to particles dispersed in $m$-cresol, but the signals are complicated (at least eight identifiable peaks) and the spectral changes due to conformational transition subtle. Only for the sake of completeness has a detailed evaluation of the FTIR evidence been added to the Supporting Information (Figure S6 and related text in Supporting Information).

Magnetic Properties of Core. The TEM images show that the cobalt particles are small

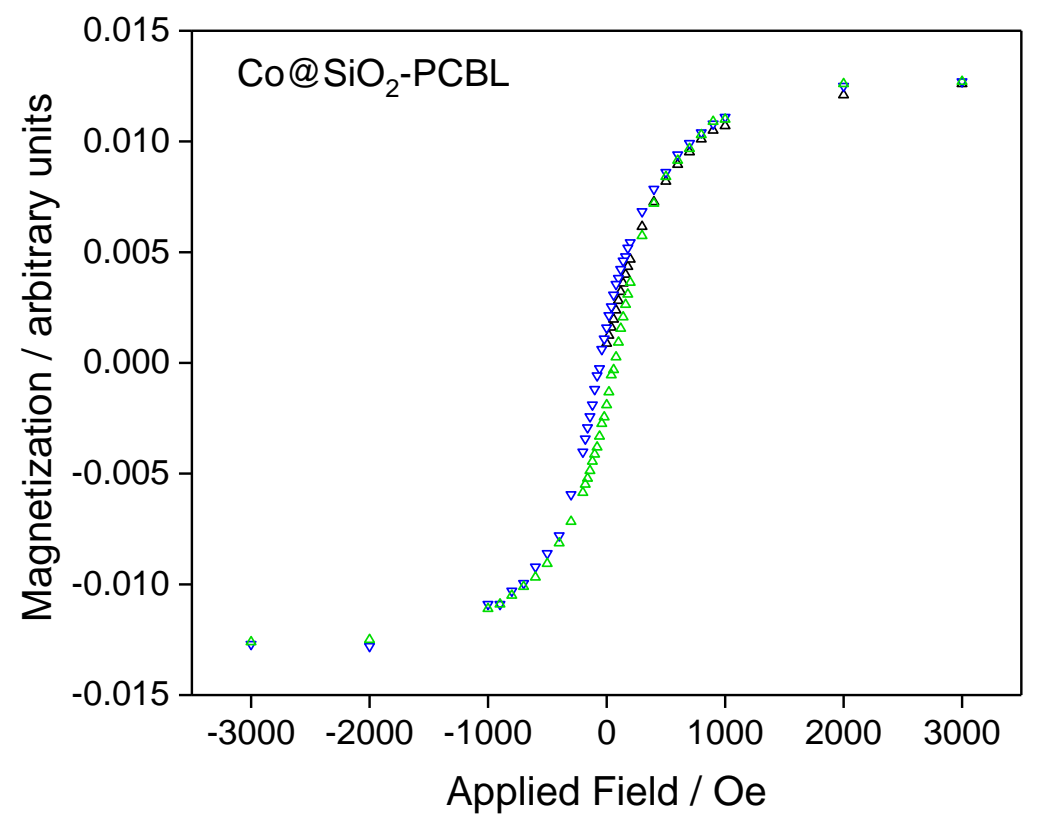

Figure 6. Magnetic hysteresis curve of dry $\mathrm{Co} @ \mathrm{SiO}_{2}-\mathrm{PCBL}$ particles in a gelatin capsule at $27^{\circ} \mathrm{C}$. Black points: initial positive field sweep from zero field; Blue points: Field sweep from 3 kOe to $-3 \mathrm{kOe}$; Green points: field sweep from $-3 \mathrm{kOe}$ to $3 \mathrm{kOe}$.

enough to exhibit superparamagnetic behavior. This feature was confirmed by SQUID measurements, as seen in S2 (Supporting Information). For comparison, the same figure also displays a magnetization curve for a commercially available superparamagnetic latex. 
Magnetic Properties of Composite Polypeptide Particle. Measurements of dry $\mathrm{Co} @ \mathrm{SiO}_{2}-\mathrm{PCBL}$ composite particles in a gelatin capsule were made at $27^{\circ} \mathrm{C}$.

As seen in Figure 6, the particles seem to exhibit a small hysteresis but we refrain from making a claim about its magnitude because apparent hysteresis can arise from the flux trapped in the superconducting magnet. Our measurements are consistent with zero hysteresis. Also, the present experiments are silent on specific magnetization of the cobalt nougat in these particles and how it changes due to the presence of a PCBL shell. Such a determination requires a difficult quantitative assessment of the small amount of cobalt in the $\mathrm{Co} @ \mathrm{SiO}_{2}$ and $\mathrm{Co} @ \mathrm{SiO}_{2}-\mathrm{PCBL}$ samples. Because PCBL mass exceeds that of the Co by a factor of about 130 (see Supporting Information) the possibility that the helix $\Leftrightarrow$ coil transition might affect the magnetic properties of the particles was considered. The magnetic susceptibility $\chi$ of PCBL-coated cobalt particles dispersed in $m$-cresol $(10 \mathrm{mg} / \mathrm{mL})$ and loaded into machined poly(tetrafluoroethylene) capsules was measured at various temperatures (Figure 7A). The particles exhibit a decreasing trend in susceptibility at $\sim 27^{\circ} \mathrm{C}$, which is the nominal $\mathrm{PCBL}$ coil $\Leftrightarrow$ helix transition temperature. As a control, bare cobalt-silica spheres were also measured in similar fashion (Figure 7B). These control particles did not demonstrate a significant change in magnetic susceptibility. The results suggest the coil $\Leftrightarrow$ helix transition slightly alters the magnetic response of the composite particles. 

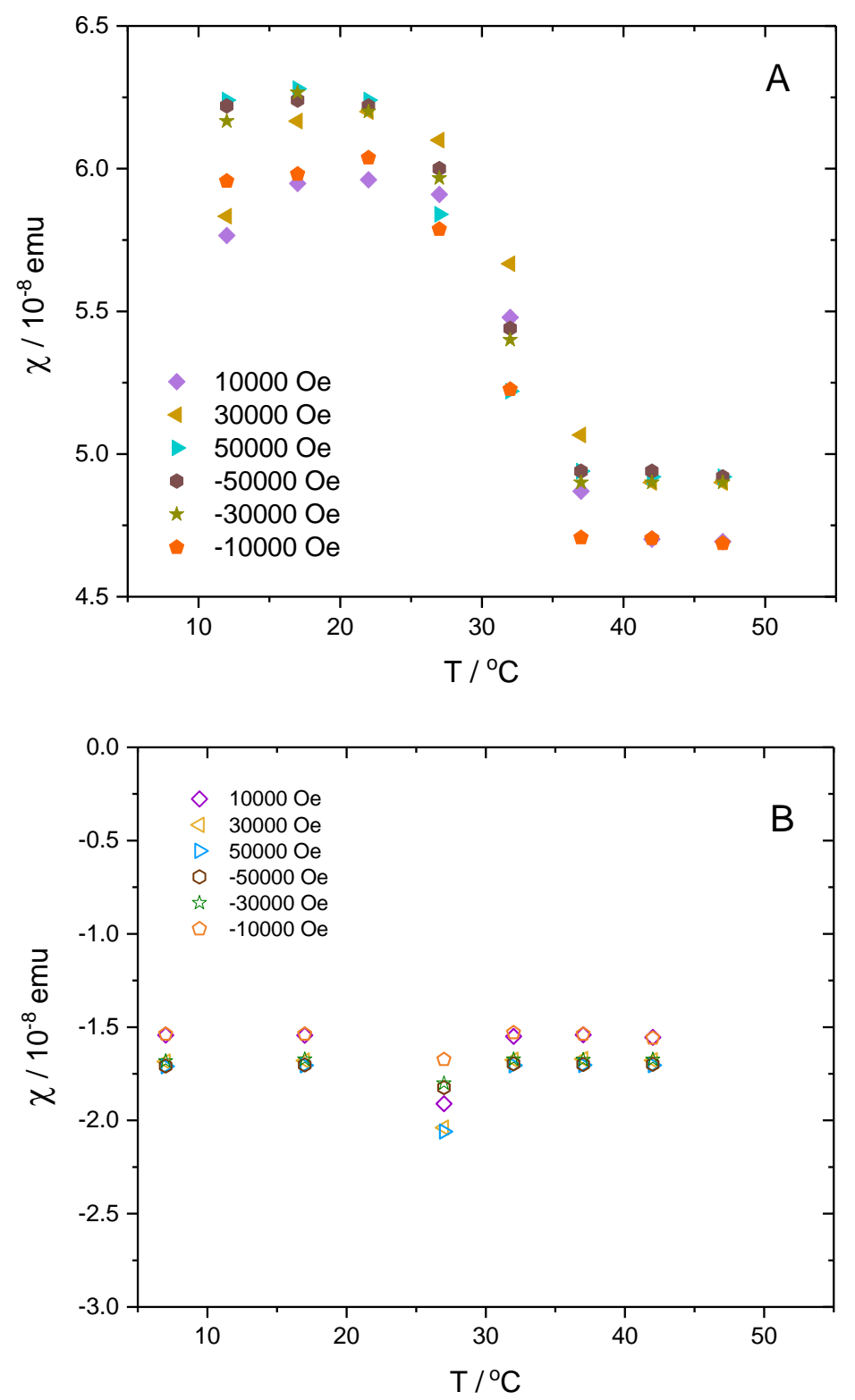

Figure 7. Magnetic susceptibility vs. temperature plots of (A) PCBL-grafted cobalt particles dispersed in $m$-cresol (for clarity, only selected fields are shown) and (B) silica-coated cobalt particles dispersed in $m$-cresol.

Aged Samples. Aging can deleteriously affect the particles in at least two ways. First, the cobalt center is susceptible to oxidation, with concomitant reduction of magnetic features such as the ability to be attracted, along with potential cargo, to a magnet. We 
do not imagine many delivery applications in $m$-cresol, but these particles should be easy to deprotect under mild conditions ${ }^{45}$ to yield poly(L-lysine)-coated magnetic silica prior to use in aqueous media where they would be expected to bind negatively charged cargo. Second, if the particles are used for fundamental studies of the tethered coil $\Leftrightarrow$ helix transition, it becomes important to know whether they must be made freshly or can be stored without loss of properties.

Both kinds of stability were assessed by re-investigating $\mathrm{Co} @ \mathrm{SiO}_{2}-\mathrm{PCBL}$ particles that had been stored in PTFE-faced screw-capped glass vials for $>5$ years. SQUID measurements showed that $\sim 50 \%$ of the initially available magnetic response remained. In contrast, Dobbrow and Schmidt, ${ }^{46}$ who studied the stability of cobalt when stabilized with polystyrene and poly(caprolactone), found the magnetic properties faded in a matter of days. In addition to protection of the Co inclusion by the silica and PCBL layers, another probable reason the $\mathrm{Co} @ \mathrm{SiO}_{2}-\mathrm{PCBL}$ particles retained more of their magnetic character is that they were suspended in $m$-cresol, which provides a sacrificial alternative target for oxygen. Visual observation supports the idea that $m$-cresol itself is not stable over such a long period; it turned a reddish color, presumably due to the formation of quinoid oxidation products. DLS studies were still possible on these reddish samples, so we had the opportunity to assess whether the PCBL shell and its transition from random coil to $\alpha$-helix when tethered to $\mathrm{Co} @ \mathrm{SiO}_{2}$ is still active after long storage. The DLS measurements revealed some aggregation at ambient temperatures (falling $R_{\mathrm{h} \text {,app }}$ vs $q^{2}$ plots) but this tendency diminished as temperature increased. For improved measurements, the particles were separated from the reddish solvent by centrifugation. The supernatant was significantly less viscous than pure $m$ cresol, suggesting the cause of the particle coalescence was slow moisture uptake during 
the long storage. When the particles were re-suspended in fresh $m$-cresol, the $R_{\mathrm{h}, \text { app }}$ vs $q^{2}$ plots flattened somewhat, but rising $\mu_{2} / \Gamma^{2}$ trends with $q^{2}$ suggested dimerization or formation of higher aggregates (so-called internal modes, including rotation, can contribute to the overall decay of correlation functions ${ }^{47}$ ). The $\mu_{2} / \Gamma^{2}$ values (typically 0.1-0.2) did not indicate wholesale aggregation, but they were higher than measured in the fresh samples ( 0.055; see Figure 4B).

What about the stability of the shell polymer, in particular the retention of a coil $\Leftrightarrow$ helix transition over time? As shown in Figure 8, aged but freshly re-dispersed particles retained their ability to expand and contract with temperature changes in $\mathrm{m}$ cresol, but the shape of the curve is as though Figure 4 were tilted slightly in the clockwise direction. The transition on heating still occurs (at slightly higher temperature, about $35^{\circ} \mathrm{C}$ ) but it follows a decline in size. After the transition, the size again decreases. This tilted curve is intriguing. Complex variations in size during helix-coil transitions have been simulated by Kemp and $\mathrm{Chen}^{48}$ for untethered polypeptides. These authors join other investigators who argue that helix-coil transitions occur in stages. Samulski, Chen and Wade enumerated some of the early evidence for multistage transitions, ${ }^{44}$ while Kastantin and Tirrell give a more recent account oriented towards transitions in polypeptide brushes. ${ }^{49}$ The broad transition evidenced in Figure 4 for fresh samples can accommodate interpretation as a gradual transition from coil to helix, 


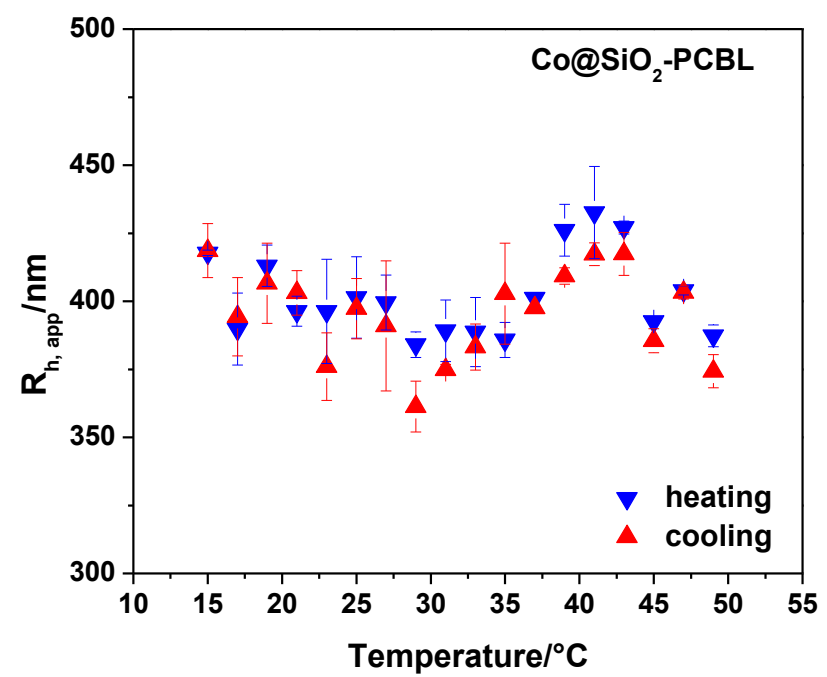

Figure 8. Apparent hydrodynamic radius vs. temperature in aged $(>5$ years) $\mathrm{Co} @ \mathrm{SiO}_{2}$-PCBL particles after redispersion in fresh $m$-cresol. Each point is an average of four heating-cooling cycles and three repeat measurements within each cycle. Measurements were performed at one angle, $173^{\circ}$ (external to the instrument).

but there is no evidence of discrete steps. The broad molecular weight distribution of the PCBL can be a factor in the breadth and continuous nature of the transition. Also, the behavior of the aged samples cannot positively be identified as related to multiple-stage conformational transitions because it is also consistent with limited aggregation that would prevent the low-temperature plateau from reaching the smallest sizes associated with fully dispersed particles. We note in passing that Yan et al. observed a non-monotonic helix-to-coil transition in their study of hollow poly(glutamate) microgels stabilized by chitosan. ${ }^{45}$ In this case, though, the 
transition is driven mostly by $\mathrm{pH}$ and is readily understood as the interplay of helical chain extension at low $\mathrm{pH}$ and charge-charge repulsion at high $\mathrm{pH}$. Summarizing this section, both functions of the $\mathrm{Co} @ \mathrm{SiO}_{2}-\mathrm{PCBL}$ particles, magnetic response and a thermally induced size transition, remain even after $>5$ years of aging but subtle changes to the thermal response were noted.

\section{CONCLUSION}

Cobalt-containing silica particles with covalently attached PCBL homopolypeptide shells, when dispersed in $m$-cresol, exhibit a broad hydrodynamic size transition as the temperature increases past $\sim 30^{\circ} \mathrm{C}$. The size change is driven by the coil $\Leftrightarrow$ helix transition of the PCBL shell polymer. PCBL was long ago was identified as a rare, thermally stimulated "shape-shifting" polypeptide that operates at a convenient temperature in a single organic solvent. ${ }^{5}$ For PCBL tethered to silica particles, the transition is reversible for a limited number of times.

The $\mathrm{Co} @ \mathrm{SiO}_{2}-\mathrm{PCBL} / m$-cresol system can serve as a model for understanding the conformational transition of tethered polymer chains without the complication of $\mathrm{pH}$ or salt effects (which nevertheless prove useful in biomaterials applications; see for example Kar et al. ${ }^{50}$ or Smith et al. ${ }^{51}$ ). It has long been thought that a two-state model for polypeptide transitions was oversimplified, ${ }^{44}$ even when the polymer chains are not tethered to a surface. For chains that are attached to a flat surface, sharp "all-or-nothing" transitions have been suggested. ${ }^{52}$ In contrast, the transitions described herein for PCBL covalently attached to a curved surface are broader than those seen by Matsuoka et al. ${ }^{5}$ for untethered PCBL in $m$-cresol, regardless of polymer molecular weight. Some of the breadth may be due to molecular weight heterogeneity. Methods sensitive to molecular structural details will be required to understand the transitions of surface-bound PCBL. 
Samples lacking the magnetic core of the present particles could be studied by NMR, for example. Such studies are relevant to the $\mathrm{pH}$-driven expansion of influenza virus during the infection process that gains them access to a host cell. ${ }^{53}$

With regard to practical applications, we envision few in $m$-cresol. This viscous, pungent and oxidatively unstable solvent was chosen specifically for the simplicity of working in a single organic solvent. Yet the opportunity to chain the particles by application of a magnetic field, followed by covalent linking to yield poly(colloids), seems promising as a route to long, responsive fibers with potential for muscle-like contraction. The stability of the magnetic core and polypeptide shell polymer suggests a long shelf life for $\mathrm{Co} @ \mathrm{SiO}_{2}$-PCBL particles and such poly(colloids) when dispersed in $m$-cresol. On an as-needed basis, it should be possible to deprotect the shell polymer to yield positively charged $\mathrm{Co}_{\mathrm{S}} \mathrm{SiO}_{2}$-poly(L-lysine) particles for magnetically directed antimicrobial formulations or delivery of negatively charged cargo such as DNA. Modulation of the magnetic response by the coil $\Leftrightarrow$ helix transition, which is an unexpected finding of this work, suggests an intriguing mechanism to control such transport operations.

\section{ACKNOWLEDGMENTS}

This material is based upon work supported by the National Science Foundation Division of Materials Research (DMR-1505105), and by a Faculty Research Grant from Louisiana State University. The magnetization and magnetic susceptibility measurements were supported by the U.S. Department of Energy under EPSCoR Grant No. DE-SC0012432 with additional support from the Louisiana Board of Regents. AS

gratefully acknowledges support through an NSF-REU Fellowship (CHE-0648841). A 
portion of this work was performed at the Georgia Tech Institute for Electronics and Nanotechnology, a member of the National Nanotechnology Coordinated Infrastructure (NNCI), which is supported by the National Science Foundation (ECCS-1542174). We are grateful for helpful suggestions from Professor Julia Chan, now at the University of Texas at Dallas, and Professor Daniel Kuroda of Louisiana State University.

Supporting Information Available. Details of DLS sample preparation, core synthesis, functionalization, characterization of commercially supplied carbobenzyloxy-L-lysine, magnetization curves, and particle characterization including XPS and TGA. This material is available free of charge via the Internet at http://pubs.acs.org. 


\section{Reference List}

1. Thanh, N. T. K.; Green, L. A. W., Functionalisation of nanoparticles for biomedical applications. Nano Today 2010, 5 (3), 213-230.

2. Tai, Y.; Wang, L.; Fan, G.; Gao, J. m.; Yu, H.; Zhang, L., Recent research progress on the preparation and application of magnetic nanospheres. Polym. Int. 2011, 60 (7), 976-994.

3. Chen, K.-J.; Chen, H.-L.; Tang, C.-C.; Wu, H.-H.; Jan, J.-S., Synthesis of silica/polypeptide hybrid nanomaterials and mesoporous silica by molecular replication of sheet-like polypeptide complexes through biomimetic mineralization. J. Colloid Interface Sci. 2019, 542, 243-252.

4. Brandenburg, B.; Koud staal, W.; Goud smit, J.; Klaren, V.; Tang, C.; Bujny, M. V.; Korse, H. J. W. M.; Kwaks, T.; Otterstrom, J. J.; Juraszek, J.; van Oijen, A. M.; Vogels, R.; Friesen, R. H. E., Mechanisms of Hemagglutinin Targeted Influenza Virus Neutralization. PLoS ONE 2013, 8 (12), e80034.

5. Matsuoka, M.; Norisuye, T.; Teramoto, A.; Fujita, H., Solution Properties of Synthetic Polypeptides. XV. Helix-Coil Transition in Poly(epsiloncarbobenzoxy L-lysine). Biopolymers 1973, 12, 1515-1532.

6. Nishioka, N.; Maekawa, A.; Teramoto, A., Solution Properties of Synthetic Polypeptides. XXI. Solvent Effect on the Helix-Coil Transition of Poly(ecarbobenzoxy L-lysine). Biopolymers 1978, 17, 665-675.

7. Omura, I.; Teramoto, A.; Fujita, H., Dielectric-Dispersion of Polypeptide Solutions .2. Helix-Coil Transition of "Poly(Epsilon-Carbobenzoxy-L-Lysine) in M-Cresol. Macromolecules 1975, 8 (3), 284-290. 
8. Karasz, F. E.; Gajnos, G. E., Thermodynamics of the Helix-Coil Transition in Polypeptides in Binary Solvent Systems. J.Phys.Chem. 1973, 77 (9), 1139-1145.

9. Poland, D.; Scheraga, H. A., Theory of Helix-Coil Transitions in Biopolymers. Academic Press: New York, 1970.

10. Wang, Y. L.; Chang, Y. C., Synthesis and conformational transition of surfacetethered polypeptide: Poly(L-lysine). Macromolecules 2003, 36 (17), 6511-6518.

11. Wang, Y. L.; Chang, Y. C., Preparation of unidirectional end-grafted alphahelical polypeptides by solvent quenching. J. Am. Chem. Soc. 2003, 125 (21), 6376-6377.

12. Wang, Y. L.; Chang, Y. C., Patterning of polypeptide thin films by the combination of surface-initiated vapor-deposition polymerization and photolithography. Advanced Materials 2003, 15 (4), 290-293.

13. Wang, Y. L.; Chang, Y. C., Grafting of homo- and block co-polypeptides on solid substrates by an improved surface-initiated vapor deposition polymerization. Langmuir 2002, 18 (25), 9859-9866.

14. Wang, Y.; Chang, Y. C., Synthesis and Conformational Transition of SurfaceTethered Polypeptide: Poly(L-glutamic acid). Macromolecules 2003, 36 (17), 6503-6510.

15. Adiga, S. P.; Brenner, D. W., Toward designing smart nanovalves: Modeling of flow control through nanopores via the helix-coil transition of grafted polypeptide chains. Macromolecules 2007, 40 (4), 1342-1348.

16. Rosu, C.; Selcuk, S.; Soto-Cantu, E.; Russo, P., Progress in silica polypeptide composite colloidal hybrids: from silica cores to fuzzy shells. Colloid Polym. Sci. 2014, 1-32. 
17. Borase, T.; Heise, A., Hybrid Nanomaterials by Surface Grafting of Synthetic Polypeptides Using N-Carboxyanhydride (NCA) Polymerization. Advanced Materials 2016, 28 (27), 5725-5731.

18. Hadjichristidis, N.; Iatrou, H.; Pitsikalis, M.; Sakellariou, G., Synthesis of welldefined polypeptide-based materials via the ring-opening polymerization of alphaamino acid N-carboxyanhydrides. Chem. Rev. 2009, 109 (11), 5528-78.

19. Huang, J.; Heise, A., Stimuli responsive synthetic polypeptides derived from Ncarboxyanhydride (NCA) polymerisation. Chem. Soc. Rev. 2013, 42 (17), 73737390.

20. Xu, Z.; Feng, Y.; Liu, X.; Guan, M.; Zhao, C.; Zhang, H., Synthesis and characterization of Fe3O4@SiO2@poly-1-alanine, peptide brush-magnetic microspheres through NCA chemistry for drug delivery and enrichment of BSA. Colloids Surf., B 2010, 81 (2), 503-507.

21. Pauling, L.; Corey, R. B., The Structure of Hair, Muscle, and Related Proteins. Proc. Natl. Acad. Sci. U.S.A. 1951, 37 (5), 261-271.

22. Liu, D.; Li, Y.; Deng, J. P.; Yang, W. T., Synthesis and characterization of magnetic Fe3O4-silica-poly(gamma-benzyl-L-glutamate) composite microspheres. React. Funct. Polym. 2011, 71 (10), 1040-1044.

23. Fong, B., unpublished results.

24. Soto-Cantu, E.; Turksen-Selcuk, S.; Qiu, J.; Zhou, Z.; Russo, P. S.; Henk, M. C., Silica-Polypeptide Composite Particles: Controlling Shell Growth. Langmuir 2010, 26 (19), 15604-15613. 
25. Turksen, S. Synthesis and Characterization of Superparamagnetic Silicahomopolypeptide Composite Particles. Chemistry, Louisiana State University, 2005.

26. Soto-Cantu, E. I. Synthesis and Surface Characterization of Silica-Polypeptide Composite Particles. 2008, Louisiana State University, 2008.

27. Rosu, C. Silica polypeptide-based colloids [electronic resource] : physical properties and novel materials. $\mathrm{PhD}$, Louisiana State University, Baton Rouge, Louisiana USA, 2013.

28. Fong, B.; Russo, P. S., Organophilic Colloidal Particles with a Synthetic Polypeptide Coating. Langmuir 1999, 15, 4421-4426.

29. Fong, B.; Turksen, S.; Russo, P. S.; Stryjewski, W., Colloidal Crystals of SilicaHomopolypeptide Composite Particles. Langmuir 2004, 20 (1), 266-269.

30. Fong, B.; Stryjewski, W.; Russo, P. S., On the Use of Pattern Fluorescence Photobleaching Recovery with Modulation Detection to Obtain Colloidal Size Distributions. J. Colloid Interface Sci. 2001, 239 (2), 374-379.

31. Soto-Cantu, E. I.; Cueto, R.; Koch, J.; Russo, P. S., Synthesis and Rapid Characterization of Amine-Functionalized Silica. Langmuir 2012, 28 (13), 55625569.

32. Kobayashi, Y.; Horie, M.; Konno, M.; Rodriguez-Gonzalez, B.; Liz-Marzan, L. M., Preparation and properties of silica-coated cobalt nanoparticles. J. Phys. Chem. B 2003, 107 (30), 7420-7425.

33. Rosu, C.; Cueto, R.; Russo, P. S., Poly(colloid)s: "Polymerization" of Poly(1tyrosine)-silica Composite Particles through the Photoinduced Cross-Linking of Unmodified Proteins Method. Langmuir 2016. 
34. Daly, W. H.; Poche', D. S., The Preparation of N-carboxyanhydrides of alphaAmino Acids Using Bis(trichloromethyl)carbonate. Tetrahedron Lett. 1988, 29(46), 5859-5862.

35. Rosu, C.; Gorman, A. J.; Cueto, R.; Dooley, K. M.; Russo, P. S., Sculpting the internal architecture of fluorescent silica particles via a template-free approach. $J$. Colloid Interface Sci. 2016, 467, 321-334.

36. Tewari, U. S.; Vesudevan, P.; Ramakrishna, V., Viscosity Behaviour of o-,m- \& p-Cresol Melts. Indian J. Chem. 1975, 13, 720-721.

37. Karasz, F. E.; Gajnos, G. E., Enthalpies of Helix-Coil Transitions in Polypeptides. Biopolymers 1974, 13 (7), 725-734.

38. Karasz, F. E.; O'Reilly, J. M., Enthalpy Changes in the Helix-Coil Transition of Poly(g-benzyl L-glutamate). Biopolymers 1967, 5, 27-35.

39. Kobayashi, Y.; Horie, M.; Konno, M.; Rodríguez-González, B.; Liz-Marzán, L. M., Preparation and Properties of Silica-Coated Cobalt Nanoparticles. J. Phys. Chem. B 2003, 107 (30), 7420-7425.

40. Block, H., Poly( g-benzyl-L-glutamate) and other Glutamic Acid Containing Polymers. Gord on and Breach: New York, 1983.

41. Fasman, G. D.; Idelson, M.; Blout, E. R., The Synthesis and Conformation of High Molecular Weight Poly-epsilon-carbobenzyloxy-L-lysine and Poly-Llysine-HCl. J.Am.Chem.Soc. 1961, 83 (Feb. 5), 709-712.

42. Doty, P.; Bradbury, J. H.; Holtzer, A. M., Polypeptides. IV. The Molecular Weight, Configuration and Association of poly(gamma-benzyl L-glutamate) in Various Solvents. J. Am. Chem. Soc. 1956, 78, 947-954. 
43. Koppel, D. E., Analysis of Macromolecular Polydispersity in Intensity Correlation Spectroscopy: The Method of Cumulants. J. Chem. Phys. 1972, 57, 4814-4820.

44. Samulski, E. T.; Chien, M.; Wade, C. G., Polypeptide sidechain secondary structure and the helix-coil transition: A NMR relaxation study. J. Polym. Sci. 1974, 46 (1), 335-340.

45. Yan, S.; Sun, Y.; Chen, A.; Liu, L.; Zhang, K.; Li, G.; Duan, Y.; Yin, J., Templated fabrication of $\mathrm{pH}$-responsive poly(l-glutamic acid) based nanogels via surface-grafting and macromolecular crosslinking. RSC Adv. 2017, 7 (24), 1488814901.

46. Dobbrow, C.; Schmidt, A. M., Improvement of the oxidation stability of cobalt nanoparticles. Beilstein J. Nanotechnol. 2012, 3, 75-81.

47. Berne, B.; Pecora, R., Dynamic Light Scattering. Wiley: New York, 1976.

48. Kemp, J. P.; Chen, J. Z. Y., Helical structures in proteins. Biomacromolecules 2001, 2 (2), 389-401.

49. Kastantin, M.; Tirrell, M., Helix Formation in the Polymer Brush.

Macromolecules 2011, 44 (12), 4977-4987.

50. Kar, M.; Pauline, M.; Sharma, K.; Kumaraswamy, G.; Sen Gupta, S., Synthesis of Poly-l-glutamic Acid Grafted Silica Nanoparticles and Their Assembly into Macroporous Structures. Langmuir 2011, 27 (19), 12124-12133.

51. Smith, I. R.; Charlier, A. H. R.; Pritzlaff, A. M.; Shishlov, A.; Barnes, B.; Bentz, K. C.; Easterling, C. P.; Sumerlin, B. S.; Fanucci, G. E.; Savin, D. A., Probing Membrane Hydration at the Interface of Self-Assembled Peptide Amphiphiles 
Using Electron Paramagnetic Resonance. ACS Macro Lett. 2018, 7 (10), 12611266.

52. Buhot, A.; Halperin, A., Extension Behavior of helicogenic polypeptides. Macromolecules 2002, 35 (8), 3238-3252.

53. Campbell, J. N.; Epand, R. M.; Russo, P. S., Structural Changes and Aggregation of Human Influenza Virus. Biomacromolecules 2004, 5 (5), 1728-1735. 
Table of Contents Graphic

For Table of Contents Use Only

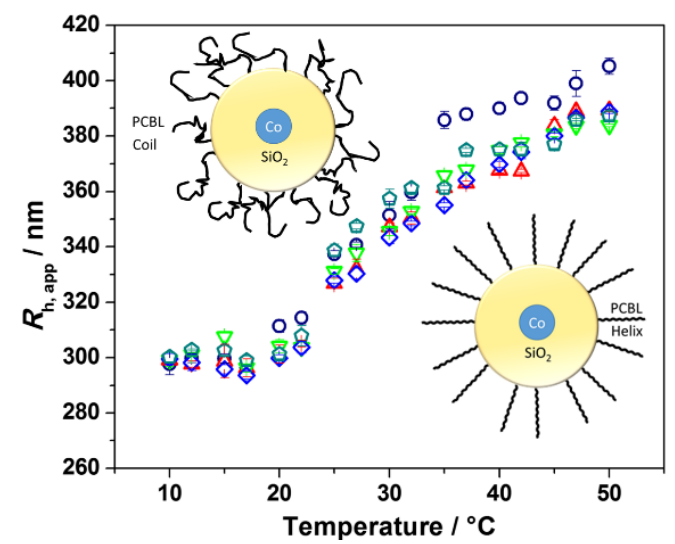

